\title{
A New Approach to the Design of a Finite Automaton that accepts Class of IPV 4 Addresses
}

\author{
P.Sri Ram Chandra ${ }^{1 *}$, K.S.Sravan ${ }^{2}$, M.S.Chakravarthy ${ }^{3}$ \\ 1, 2, 3 Department of Computer Science and Engineering, Godavari Institute of Engineering and Technology (A), \\ Rajahmundry, Andhra Pradesh, India.
}

Received: 19 April 2018; Accepted: 06 August 2018; Published: 08 January 2019

\begin{abstract}
Theory of computation is characterized as calculation through the conceptual machines. The three essential unique machines utilized are Finite Automata, Pushdown Automata and Turing Machine. In this paper we propose an outline of Finite Automata that accepts the class of $\mathrm{IPV}_{4}$ Addresses.
\end{abstract}

Index Terms: Finite Automata, IP Address.

(C) 2019 Published by MECS Publisher. Selection and/or peer review under responsibility of the Research Association of Modern Education and Computer Science

\section{Introduction}

Theory of computation is defined as computation through the abstract machines. Finite Automata is one among the primary abstract machines. It is represented as a five tuple machine ' $M$ '.

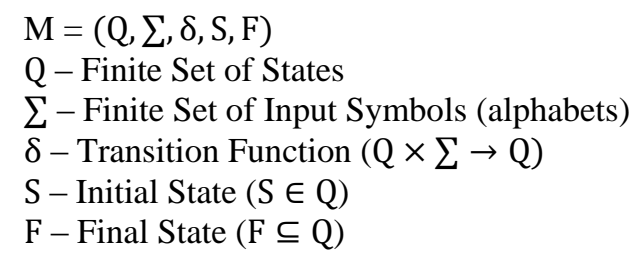

* Corresponding author.

E-mail address: psrgietcse@gmail.com 


\subsection{Alphabets}

An alphabet is a finite, nonempty set of inputs usually denoted as $\sum$.

Examples of the alphabets

- Binary alphabet $\sum=\{0,1\}$

- English alphabet $\sum=\{a, b, \ldots, z\}$

\section{Strings}

A string (or word) is a finite sequence of symbols from an alphabet. For example --- 1011 is a string from the binary alphabet $\sum=\{0,1\}$.

- Empty string ' 'is defined as a string with zero occurrences of symbols.

- Length ' $|\mathrm{W}|$ ' of string ' $\mathrm{W}$ ' is defined as the number of positions for symbols in W. For example length of the string ' 0111 ' is $|0111|=4$, Length of the string ' ' is $|\quad|=0$.

- Set of all strings over $\sum$ is usually denoted as $\sum^{*}$ i.e., $\Sigma^{*}=\sum^{0} U \sum^{1} U^{* 2} U \ldots \Sigma^{n}$

- $\Sigma^{+}=$the set of nonempty strings from $\sum=\Sigma^{*}-\{\quad\}$. Therefore, we have

$$
\Sigma^{+}=\sum^{1} \mathbf{U} \Sigma^{2} \mathbf{U} \Sigma^{3} \mathbf{U} \ldots \sum^{\mathrm{n}} \text { and } \Sigma^{*}=\Sigma^{+} \mathbf{U}\{\varepsilon\}
$$

\section{Languages}

A language is a set of strings all chosen from some $\sum^{*}$. In other words, if $\sum$ is an alphabet, and $\mathrm{L} \subseteq \sum^{*}$, then $\mathrm{L}$ is a language over $\sum$.

Examples:

- For the language L: Set of all legal English words, $\sum=$ the set of all letters $\}$

- For the language L: Program written in ' $\mathrm{C}$ ' Language, $\sum=\{$ a subset of the ASCII characters $\}$

- The set of all strings of $\mathrm{n}$ 0's followed by $\mathrm{n}$ 1's for $\mathrm{n} \geq 0$ : $\{\varepsilon, 01,0011,000111, \ldots\}$

- $\sum^{*}$ is an infinite language for any alphabet $\sum$.

- $\varnothing$ Denotes the empty language (not the empty string $\varepsilon$ ) which is a language over any alphabet.

- $\{\varepsilon\}$ is a language over any alphabet (consisting of only one string, the empty string $\varepsilon$ ).

\section{Different ways of describing languages}

\section{Description by exhaustive listing}

- $\mathrm{L}_{1}=\{\mathrm{a}, \mathrm{ab}, \mathrm{abc}\}$ (finite language; listed one by one)

- $\mathrm{L}_{2}=\{\mathrm{a}, \mathrm{ab}, \mathrm{abb}, \mathrm{abbb}, \ldots\}$ (infinite language; listed partially)

- $\mathrm{L}_{3}=\mathrm{L}\left(\mathrm{ab}{ }^{*}\right)$ (infinite language; expressed by a regular expression)

\section{Description by generic elements}

- $\mathrm{L}_{4}=\{\mathrm{x} \mid \mathrm{x}$ is over $\mathrm{V}=\{\mathrm{a}, \mathrm{b}\}$, begins with $\mathrm{a}$, followed by any number of $\mathrm{b}$, possible none $\}$

. Note: $\mathrm{L}_{4}=\mathrm{L}_{3}=\mathrm{L}_{2}$ 


\section{Description by integer parameters}

- $\mathrm{L}_{5}=\left\{\mathrm{ab}^{\mathrm{n}} \mid \mathrm{n} \geq 0\right\}$

- Note: $\mathrm{L}_{5}=\mathrm{L}_{4}=\mathrm{L}_{3}=\mathrm{L}_{2}$

\section{Closures of language $\mathrm{L}$ :}

- Positive closure of a language $\mathrm{L}$ is usually denoted as $\mathrm{L}^{+}=\mathrm{L}^{1} \mathrm{UL}{ }^{2} \mathrm{U} \ldots \mathrm{L}^{\mathrm{n}}$

- Star Closure of a language $\mathrm{L}$ is usually denoted as $\mathrm{L}^{*}=\bigcup_{i=0}^{\infty} L^{i}=\mathrm{L}^{0} \mathrm{UL}^{1} \mathrm{UL} \mathrm{L}^{2} \mathrm{U} \ldots \mathrm{L}^{\mathrm{n}}$

- Note: $\mathrm{L}^{*}-\mathrm{L}^{0}=\mathrm{L}^{*}-\{\varepsilon\}$

\subsection{Internet Protocol (IP) Addresses and Automata}

The key contribution to this research paper starts with the basic idea about IPV $_{4}$ addresses and identifying the required relationships between $\mathrm{IPV}_{4}$ address and Constructing Finite Automata. Usually the communication between the computers can be done through the network. For any level of communication we need a global addressing scheme i.e., IP addressing scheme to mean a logical address in the network layer of the TCP/IP protocol suite. These addresses are referred to as IPV ${ }_{4}$ addresses. An IPV 4 address is a 32 bit address that uniquely defines the connection of a device through Internet. The address space of the IPV $\mathrm{Iddress}_{4}$ is $2^{32}$

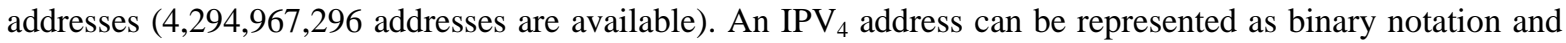

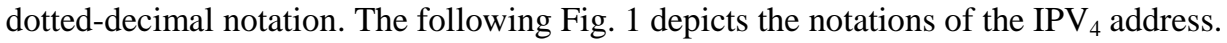

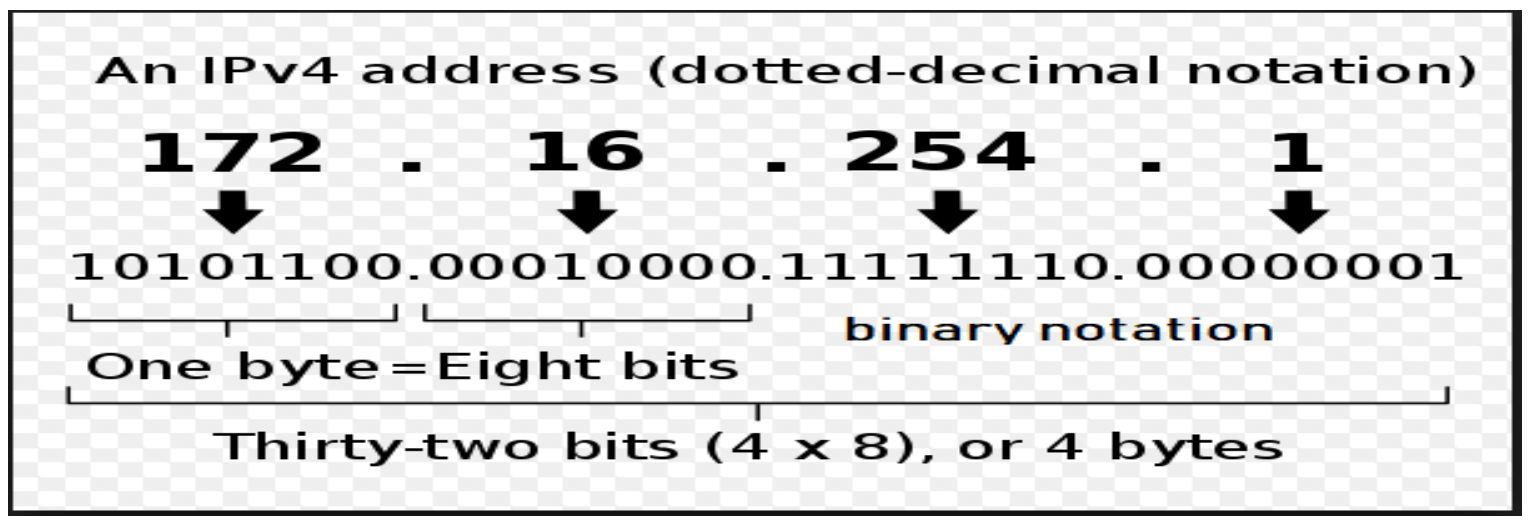

Fig.1. Binary and Dotted-Decimal Notations of the $\mathrm{IPV}_{4}$ address.

\subsection{Classful Addressing}

Since its inception, the IPV $\mathrm{V}_{4}$ addressing used the concept of classes. In classful addressing, the address space is divided into five classes namely A, B, C, D and E. Each class occupies some part of the address space. We can find the class of an address when it is represented in binary or dotted-decimal notations. The first few bits in binary notation of IP address will tell us the class name. The first byte in dotted-decimal notation of IP address also tells us the class. The following Fig. 2 depicts both the methods. As far as the finite automaton is concerned we use only binary notation of IP address to detect its class. 


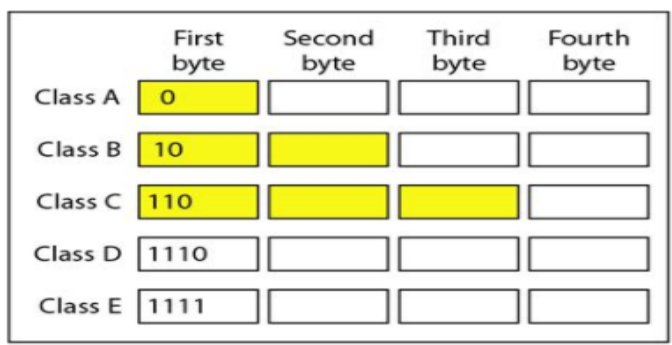

a. Binary notation

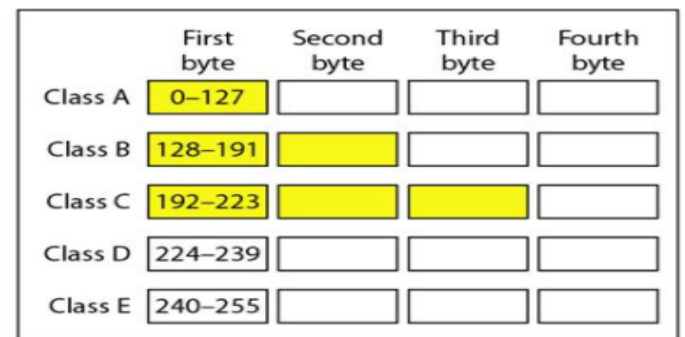

b. Dotted-decimal notation

Fig.2. Methods to detect the class of IP Address

The Fig. 2 clearly depicts that, first few bits in binary notation of IPV $\mathrm{V}_{4}$ address plays a vital role in detecting its respective class. As the first bit (MSB) of Class A in Binary notation is ' 0 ', we can say that it is the string of 32 bits [actual length of $\mathrm{IPV}_{4}$ address]. According to the Theory of Automata, the language for the Class A address is designated as $\mathrm{L}=\left\{0 \mathrm{~W} \mid \mathrm{W} \in(0+1)^{*}\right\}$. Similarly the language for rest of the classes is as follows:

Language to detect Class B IP address: $\mathrm{L}=\{10 \mathrm{~W} \mid \mathrm{W} \in(0+1) *\}$

Language to detect Class C IP address: $\mathrm{L}=\left\{110 \mathrm{~W} \mid \mathrm{W} \in(0+1)^{*}\right\}$

Language to detect Class D IP address: $\mathrm{L}=\{1110 \mathrm{~W} \mid \mathrm{W} \in(0+1) *\}$

Language to detect Class E IP address: $\mathrm{L}=\left\{1111 \mathrm{~W} \mid \mathrm{W} \in(0+1)^{*}\right\}$

\section{Construction of Finite Automata}

In this section we describe the construction of Finite Automata based on the corresponding languages defined earlier.

\subsection{Design of a Finite Automata to accept Class 'A' IP address}

According to the Fig. 2, the first bit of Class A in Binary notation is ' 0 ', which indicates that it is a string of 32 bits [actual length of IPV4 address]. Thus the language for the Class ' $A$ ' address is designated as

$$
\mathrm{L}=\{0 \mathrm{~W} \mid \mathrm{W} \in(0+1) *\}
$$

or

$\mathrm{L}=\{0$ followed by any number of 0 s and any number of $1 \mathrm{~s}\}$.

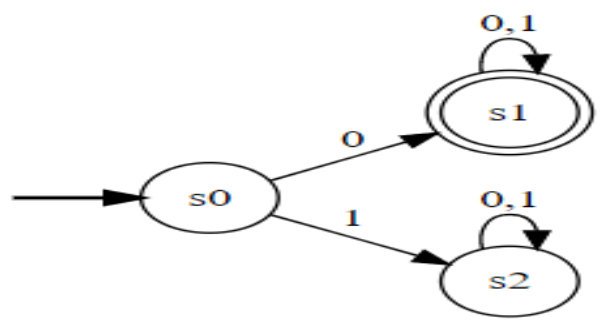

Fig.3. Transition Diagram of a Finite Automata the accepts the Class 'A' IP Addresses 
The input alphabets are denoted as $\sum=\{0,1\}$ and the corresponding Regular Expression is given as: $0(0+1)^{*}$. The following Fig. 3 depicts the corresponding automata.

Finite Automata are often represented by digraphs called transition diagram. The vertices (denoted by single circles) of a transition diagram represents various states of the Finite Automata and the edges labelled with an input symbol correspond to the transitions. An edge $(0,1)$ from state $s_{0}$ to state $s_{1}$ with label ' 0 ' represents the transition $\delta\left(s_{0}, 0\right)=s_{1}$. The acceptance states or final states are depicted by double circles. Transition functions can also be represented by tables as shown in the table1called as transition table.

Table 1. Transition Table for the Finite Automata shown in Fig. 3

\begin{tabular}{c|cc}
\hline & \multicolumn{2}{|c}{$\sum$} \\
\cline { 2 - 3 }$Q$ & 0 & 1 \\
\hline $\mathrm{s}_{0}$ & $\mathrm{~s}_{1}$ & $\mathrm{~s}_{2}$ \\
$\mathrm{~s}_{1}$ & $\mathrm{~s}_{1}$ & $\mathrm{~s}_{1}$ \\
$\mathrm{~s}_{2}$ & $\mathrm{~s}_{2}$ & $\mathrm{~s}_{2}$ \\
\hline
\end{tabular}

\subsection{Design of a Finite Automata to accept Class 'B' IP address}

In the Fig. 2, the first two bits of Class ' $\mathrm{B}$ ' in Binary notation are ' 10 ', means that it is the string of 32 bits [actual length of IPV4 address] where the two bits from MSB (including) are confined to '10'. Thus the language for the Class B address is designated as

$$
\mathrm{L}=\left\{10 \mathrm{~W} \mid \mathrm{W} \in(0+1)^{*}\right\}
$$

or

$$
L=\{10 \text { followed by any number of } 0 \text { s and any number of } 1 s\} .
$$

The input alphabets are denoted as $\sum=\{0,1\}$ and the corresponding Regular Expression is given as: $10(0+1) *$. The following Fig. 4 depicts the corresponding automata.

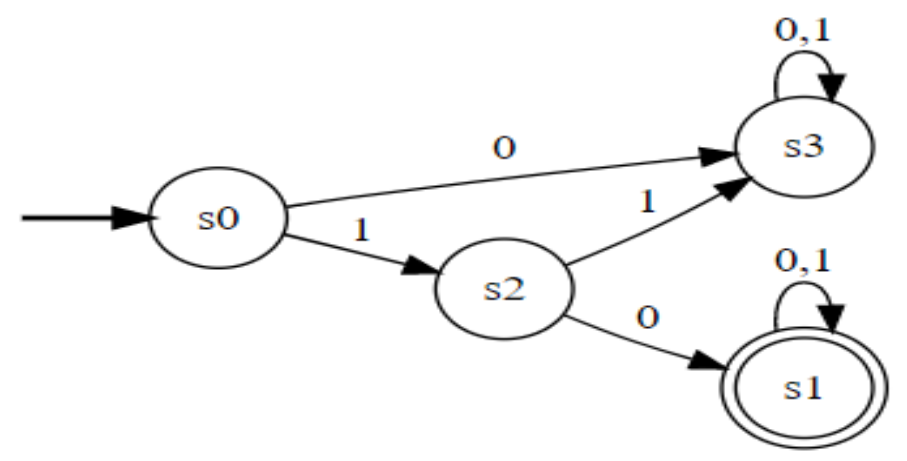

Fig.4 Transition Diagram of a Finite Automata the accepts the Class 'B' IP Addresses 
Table 2. Transition Table for the Finite Automata shown in Fig. 4

\begin{tabular}{c|cc}
\hline \multirow{2}{*}{$Q$} & \multicolumn{2}{|c}{$\sum$} \\
\cline { 2 - 3 } & $\mathbf{0}$ & $\mathbf{1}$ \\
\hline $\mathbf{s}_{\mathbf{0}}$ & $\mathbf{s}_{3}$ & $\mathbf{s}_{2}$ \\
$\mathbf{s}_{1}$ & $\mathbf{s}_{1}$ & $\mathbf{s}_{1}$ \\
$\mathbf{s}_{2}$ & $\mathbf{s}_{1}$ & $\mathbf{s}_{3}$ \\
$\mathbf{s}_{3}$ & $\mathbf{s}_{3}$ & $\mathbf{s}_{3}$ \\
\hline
\end{tabular}

\subsection{Design of a Finite Automata to accept Class ' $C$ ' IP address}

As shown in the Fig. 2, the first two bits of Class C in Binary notation are '110', means that it is a string of 32 bits [actual length of IPV4 address] where the three bits from MSB are confined to ' 110 '. Thus the language for the Class $\mathrm{C}$ address is designated as

$$
\mathrm{L}=\left\{110 \mathrm{~W} \mid \mathrm{W} \in(0+1)^{*}\right\}
$$

or

$$
\mathrm{L}=\{110 \text { followed by any number of } 0 \text { s and any number of } 1 \mathrm{~s}\}
$$

The input alphabets are denoted as $\sum=\{0,1\}$ and the corresponding Regular Expression is given as: $110(0+1) *$. The following Fig. 5 depicts the corresponding automata.

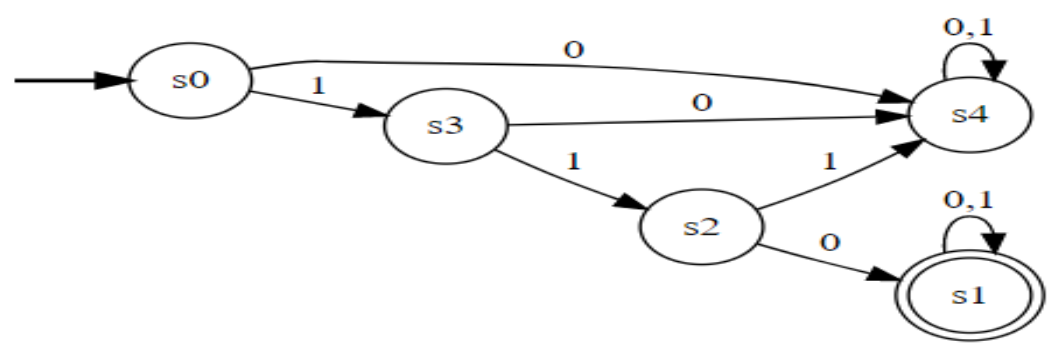

Fig.5. Transition Diagram of a Finite Automata the accepts the Class 'C' IP Addresses

Table 3. Transition Table for the Finite Automata shown in Fig. 5

\begin{tabular}{l|ll}
\hline & \multicolumn{2}{|c}{$\sum$} \\
\cline { 2 - 3 } $\mathbf{Q}$ & $\mathbf{0}$ & $\mathbf{1}$ \\
$\mathbf{s}_{0}$ & $\mathbf{s}_{4}$ & $\mathbf{s}_{3}$ \\
$\mathbf{s}_{1}$ & $\mathbf{s}_{1}$ & $\mathbf{s}_{1}$ \\
$\mathbf{s}_{2}$ & $\mathbf{s}_{1}$ & $\mathbf{s}_{4}$ \\
$\mathbf{s}_{3}$ & $\mathbf{s}_{4}$ & $\mathbf{s}_{2}$ \\
$\mathbf{s}_{4}$ & $\mathbf{s}_{4}$ & $\mathbf{s}_{4}$ \\
\hline
\end{tabular}




\subsection{Design of a Finite Automata to accept Class 'D' IP address}

As shown in the Fig. 2, the first two bits of Class D in Binary notation are '1110', means that it is a string of 32 bits [actual length of IPV4 address] where the four bits from MSB are confined to ' 1110 '. Thus the language for the Class D address is designated as

$$
\mathrm{L}=\left\{1110 \mathrm{~W} \mid \mathrm{W} \in(0+1)^{*}\right\}
$$

or

$$
\mathrm{L}=\{1110 \text { followed by any number of } 0 \text { s and any number of } 1 \mathrm{~s}\} .
$$

The input alphabets are denoted as $\sum=\{0,1\}$ and the corresponding Regular Expression is given as: $1110(0+1)^{*}$. The following Fig. 5 depicts the corresponding automata.

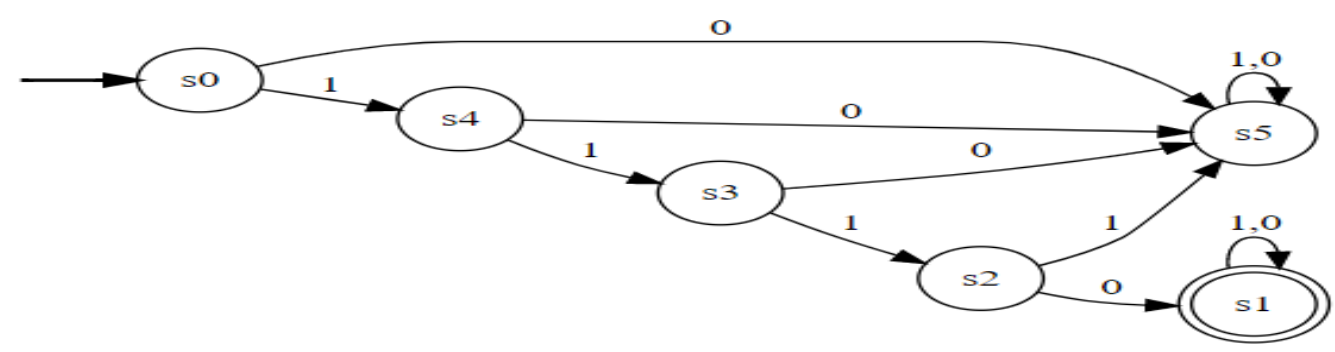

Fig.6. Transition Diagram of a Finite Automata the accepts the Class 'D' IP Addresses

Table 4. Transition Table for the Finite Automata shown in Fig. 6

\begin{tabular}{c|cc}
\hline & \multicolumn{2}{|c}{$\sum$} \\
\cline { 2 - 3 }$Q$ & 0 & 1 \\
\hline $\mathrm{s}_{0}$ & $\mathrm{~s}_{5}$ & $\mathrm{~s}_{4}$ \\
$\mathrm{~s}_{1}$ & $\mathrm{~s}_{1}$ & $\mathrm{~s}_{1}$ \\
$\mathrm{~s}_{2}$ & $\mathrm{~s}_{1}$ & $\mathrm{~s}_{5}$ \\
$\mathrm{~s}_{3}$ & $\mathrm{~s}_{5}$ & $\mathrm{~s}_{2}$ \\
$\mathrm{~s}_{4}$ & $\mathrm{~s}_{5}$ & $\mathrm{~s}_{3}$ \\
$\mathrm{~s}_{5}$ & $\mathrm{~s}_{5}$ & $\mathrm{~s}_{1}$ \\
\hline
\end{tabular}

\subsection{Design of a Finite Automata to accept Class 'E' IP address}

As shown in the Fig. 2, the first two bits of Class E in Binary notation are '1111', means that it is a string of 32 bits [actual length of IPV4 address] where the four bits from MSB are confined to '1111'. Thus the language for the Class E address is designated as 


$$
\mathrm{L}=\{1111 \mathrm{~W} \mid \mathrm{W} \in(0+1) *\}
$$

or

$$
\mathrm{L}=\{1111 \text { followed by any number of } 0 \text { s and any number of } 1 \mathrm{~s}\} .
$$

The input alphabets are denoted as $\sum=\{0,1\}$ and the corresponding Regular Expression is given as: $1110(0+1)^{*}$. The following Fig. 5 depicts the corresponding automata.

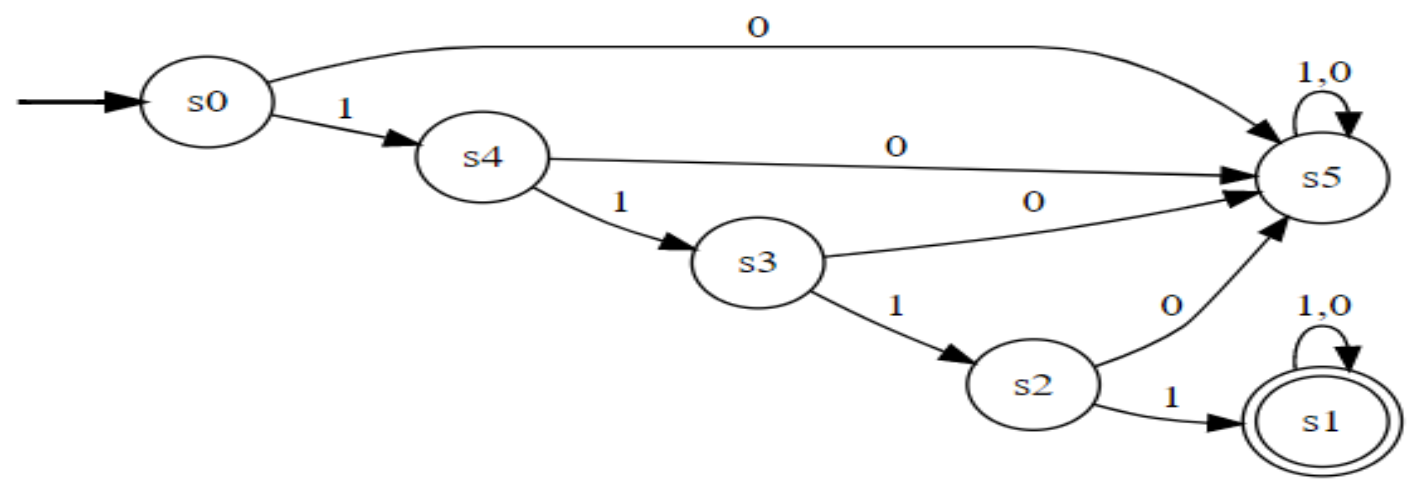

Fig.7. Transition Diagram of a Finite Automata the accepts the Class 'E' IP Addresses

\begin{tabular}{|c|c|c|}
\hline & \multicolumn{2}{|c|}{$\sum$} \\
\hline $\mathbf{Q}$ & 0 & 1 \\
\hline $\mathbf{s}_{0}$ & $\mathbf{S}_{5}$ & $\mathbf{s}_{4}$ \\
\hline $\mathbf{s}_{1}$ & $\mathbf{s}_{1}$ & $\mathbf{s}_{1}$ \\
\hline $\mathbf{S}_{2}$ & $\mathbf{S}_{5}$ & $\mathbf{s}_{1}$ \\
\hline $\mathbf{S}_{\mathbf{3}}$ & $\mathbf{S}_{5}$ & $\mathbf{S}_{2}$ \\
\hline $\mathbf{s}_{4}$ & $\mathbf{S}_{5}$ & $\mathbf{S}_{3}$ \\
\hline $\mathbf{S}_{5}$ & $\mathbf{S}_{5}$ & $\mathbf{s}_{1}$ \\
\hline
\end{tabular}

Table 5. Transition Table for the Finite Automata shown in Fig. 7

\section{Results and Discussion}

As far as the results section is concerned, we need to prove that the designed automata should accept the respective class of IP address as per the language mentioned. We can say that the automaton accepts the respective class of IP address if and only if "the validation of the considered 32 bit string starts at initial state and ends at final state". At first we will see the validation for Class ' $A$ ' $I P V_{4}$ addresses that is 32 bit string with MSB as ' 0 ', further we will see the rest of classes.

The input string to validate class ' $A$ ' IP Address: 


\begin{tabular}{|c|c|c|c|}
\hline & \multirow{2}{*}{ Table 6.a } & Dotted Decimal Notation & [Should be Accepted] \\
\hline \multirow{3}{*}{$\begin{array}{c}\mathrm{IPV}_{4} \\
\text { Address }\end{array}$} & & Binary Notation & 01001111011011110111111101100011 \\
\hline & \multirow{2}{*}{ Table 6.b } & Dotted Decimal Notation & [Should be Rejected] \\
\hline & & Binary Notation & 11001111011011110100010101100110 \\
\hline
\end{tabular}

In the table 6.a, the last state achieved through the automata is a final state. The automata is verified and hence proved that it accepts all the strings which start with '0'i.e., Class 'A' IPV4 Address. In the table 6.b, since the last state obtained is $\mathrm{s} 2$ and as it is not the final state in the automata, so the strings which start with other than ' 0 ' are not accepted i.e., it accepts only Class 'A' IPV4 Address.

Table 6. Validation Table that shows the automata accepts Class a IPV 4 Address

\begin{tabular}{|c|c|c|c|c|c|c|c|c|c|c|c|}
\hline \multicolumn{6}{|c|}{ Table 6.a } & \multicolumn{6}{|c|}{ Table 6.b } \\
\hline $\begin{array}{l}\text { Current } \\
\text { State-Q }\end{array}$ & Input- $\Sigma$ & $\begin{array}{c}\text { Next } \\
\text { State- } \\
\text { Q }\end{array}$ & $\begin{array}{l}\text { Current } \\
\text { State-Q }\end{array}$ & Input- $\Sigma$ & $\begin{array}{c}\text { Next } \\
\text { State- } \\
\text { Q }\end{array}$ & $\begin{array}{l}\text { Current } \\
\text { State-Q }\end{array}$ & Input- $\Sigma$ & $\begin{array}{c}\text { Next } \\
\text { State- } \\
\text { Q }\end{array}$ & $\begin{array}{l}\text { Current } \\
\text { State-Q }\end{array}$ & Input- $\Sigma$ & $\begin{array}{c}\text { Next } \\
\text { State-Q }\end{array}$ \\
\hline \multicolumn{3}{|c|}{$\mathrm{Q} X \Sigma \rightarrow \mathrm{Q}$} & \multicolumn{3}{|c|}{$\mathrm{Q} X \Sigma \rightarrow \mathrm{Q}$} & \multicolumn{3}{|c|}{$\mathrm{Q} X \sum \rightarrow \mathrm{Q}$} & \multicolumn{3}{|c|}{$\mathrm{Q} X \sum \rightarrow \mathrm{Q}$} \\
\hline $\mathrm{s}_{0}$ & 0 & $\mathrm{~s}_{1}$ & $\mathrm{~s}_{1}$ & 0 & $\mathrm{~s}_{1}$ & $\mathrm{~s}_{0}$ & 1 & $\mathrm{~s}_{2}$ & $\mathrm{~s}_{2}$ & 0 & $\mathrm{~s}_{2}$ \\
\hline $\mathrm{s}_{1}$ & 1 & $\mathrm{~s}_{1}$ & $\mathrm{~s}_{1}$ & 1 & $\mathrm{~s}_{1}$ & $\mathrm{~S}_{2}$ & 1 & $\mathrm{~s}_{2}$ & $\mathrm{~s}_{2}$ & 1 & $\mathrm{~s}_{2}$ \\
\hline $\mathrm{s}_{1}$ & 0 & $\mathrm{~s}_{1}$ & $\mathrm{~s}_{1}$ & 1 & $\mathrm{~s}_{1}$ & $\mathrm{~S}_{2}$ & 0 & $\mathrm{~s}_{2}$ & $\mathrm{~s}_{2}$ & 0 & $\mathrm{~s}_{2}$ \\
\hline $\mathrm{s}_{1}$ & 0 & $\mathrm{~s}_{1}$ & $\mathrm{~s}_{1}$ & 1 & $\mathrm{~s}_{1}$ & $\mathrm{~s}_{2}$ & 0 & $\mathrm{~s}_{2}$ & $\mathrm{~s}_{2}$ & 0 & $\mathrm{~s}_{2}$ \\
\hline $\mathrm{s}_{1}$ & 1 & $\mathrm{~s}_{1}$ & $\mathrm{~s}_{1}$ & 1 & $\mathrm{~s}_{1}$ & $\mathrm{~s}_{2}$ & 1 & $\mathrm{~S}_{2}$ & $\mathrm{~s}_{2}$ & 0 & $\mathrm{~s}_{2}$ \\
\hline $\mathrm{s}_{1}$ & 1 & $\mathrm{~s}_{1}$ & $\mathrm{~s}_{1}$ & 1 & $\mathrm{~s}_{1}$ & $\mathrm{~s}_{2}$ & 1 & $\mathrm{~s}_{2}$ & $\mathrm{~s}_{2}$ & 1 & $\mathrm{~s}_{2}$ \\
\hline $\mathrm{s}_{1}$ & 1 & $\mathrm{~s}_{1}$ & $\mathrm{~s}_{1}$ & 1 & $\mathrm{~s}_{1}$ & $\mathrm{~s}_{2}$ & 1 & $\mathrm{~s}_{2}$ & $\mathrm{~s}_{2}$ & 0 & $\mathrm{~s}_{2}$ \\
\hline $\mathrm{s}_{1}$ & 1 & $\mathrm{~s}_{1}$ & $\mathrm{~s}_{1}$ & 1 & $\mathrm{~s}_{1}$ & $\mathrm{~s}_{2}$ & 1 & $\mathrm{~s}_{2}$ & $\mathrm{~s}_{2}$ & 1 & $\mathrm{~s}_{2}$ \\
\hline $\mathrm{s}_{1}$ & 0 & $\mathrm{~s}_{1}$ & $\mathrm{~s}_{1}$ & 0 & $\mathrm{~s}_{1}$ & $\mathrm{~s}_{2}$ & 0 & $\mathrm{~s}_{2}$ & $\mathrm{~s}_{2}$ & 0 & $\mathrm{~s}_{2}$ \\
\hline $\mathrm{s}_{1}$ & 1 & $\mathrm{~s}_{1}$ & $\mathrm{~s}_{1}$ & 1 & $\mathrm{~s}_{1}$ & $\mathrm{~s}_{2}$ & 1 & $\mathrm{~s}_{2}$ & $\mathrm{~s}_{2}$ & 1 & $\mathrm{~s}_{2}$ \\
\hline $\mathrm{s}_{1}$ & 1 & $\mathrm{~s}_{1}$ & $\mathrm{~s}_{1}$ & 1 & $\mathrm{~s}_{1}$ & $\mathrm{~s}_{2}$ & 1 & $\mathrm{~s}_{2}$ & $\mathrm{~s}_{2}$ & 1 & $\mathrm{~s}_{2}$ \\
\hline $\mathrm{s}_{1}$ & 0 & $\mathrm{~s}_{1}$ & $\mathrm{~s}_{1}$ & 0 & $\mathrm{~s}_{1}$ & $\mathrm{~s}_{2}$ & 0 & $\mathrm{~s}_{2}$ & $\mathrm{~s}_{2}$ & 0 & $\mathrm{~s}_{2}$ \\
\hline $\mathrm{s}_{1}$ & 1 & $\mathrm{~s}_{1}$ & $\mathrm{~s}_{1}$ & 0 & $\mathrm{~s}_{1}$ & $\mathrm{~s}_{2}$ & 1 & $\mathrm{~s}_{2}$ & $\mathrm{~s}_{2}$ & 0 & $\mathrm{~s}_{2}$ \\
\hline $\mathrm{s}_{1}$ & 1 & $\mathrm{~s}_{1}$ & $\mathrm{~s}_{1}$ & 0 & $\mathrm{~s}_{1}$ & $\mathrm{~s}_{2}$ & 1 & $\mathrm{~s}_{2}$ & $\mathrm{~s}_{2}$ & 1 & $\mathrm{~s}_{2}$ \\
\hline $\mathrm{s}_{1}$ & 1 & $\mathrm{~s}_{1}$ & $\mathrm{~s}_{1}$ & 1 & $\mathrm{~s}_{1}$ & $\mathrm{~s}_{2}$ & 1 & $\mathrm{~s}_{2}$ & $\mathrm{~s}_{2}$ & 1 & $\mathrm{~s}_{2}$ \\
\hline $\mathrm{s}_{1}$ & 1 & $\mathrm{~s}_{1}$ & $\mathrm{~s}_{1}$ & 1 & $\mathrm{~s}_{1}$ & $\mathrm{~s}_{2}$ & 1 & $\mathrm{~s}_{2}$ & $\mathrm{~s}_{2}$ & 0 & $\mathrm{~s}_{2}$ \\
\hline
\end{tabular}


The input string to validate class 'B' IP Address:

\begin{tabular}{|c|c|l|l|}
\hline & & & \\
\hline \multirow{2}{*}{$\begin{array}{c}\mathrm{IPV}_{4} \\
\text { Address }\end{array}$} & Table 7.a & Dotted Decimal Notation & $143.111 .127 .99 \quad[$ Should be Accepted] \\
\cline { 3 - 5 } & & Binary Notation & 100011110110111101111111101100011 \\
\cline { 3 - 5 } & \multirow{2}{*}{ Table 7.b } & Dotted Decimal Notation & $207.111 .69 .102 \quad[$ Should be Rejected] \\
\cline { 3 - 5 } & & Binary Notation & 11001111011011110100010101100110 \\
\hline
\end{tabular}

In the table 7.a, the last state achieved through the automata is a final state. The automata is verified and hence proved that it accepts all the strings which start with ' 10 'i.e., Class ' $\mathrm{B}$ ' IPV 4 Address. In the table 7.b, since the last state obtained is $\mathrm{s}_{3}$ and as it is not the final state in the automata, so the strings which start with other than ' 10 ' are not accepted i.e., it accepts only Class 'B' IPV 4 Address.

Table 7. Validation table that shows the Automata accepts Class 'B' IPV4 Address

\begin{tabular}{|c|c|c|c|c|c|c|c|c|c|c|c|}
\hline \multicolumn{6}{|c|}{ Table 7.a } & \multicolumn{6}{|c|}{ Table 7.b } \\
\hline $\begin{array}{l}\text { Current } \\
\text { State-Q }\end{array}$ & $\begin{array}{l}\text { Input- } \\
\sum\end{array}$ & $\begin{array}{c}\text { Next } \\
\text { State-Q }\end{array}$ & $\begin{array}{l}\text { Current } \\
\text { State-Q }\end{array}$ & $\stackrel{\text { Input- }}{\sum}$ & $\begin{array}{c}\text { Next } \\
\text { State-Q }\end{array}$ & $\begin{array}{l}\text { Current } \\
\text { State-Q }\end{array}$ & $\begin{array}{l}\text { Input- } \\
\sum\end{array}$ & $\begin{array}{c}\text { Next } \\
\text { State-Q }\end{array}$ & $\begin{array}{l}\text { Current } \\
\text { State-Q }\end{array}$ & $\begin{array}{l}\text { Input- } \\
\sum\end{array}$ & $\begin{array}{c}\text { Next } \\
\text { State-Q }\end{array}$ \\
\hline \multicolumn{3}{|c|}{$\mathrm{Q} X \quad \sum \rightarrow \mathrm{Q}$} & \multicolumn{3}{|c|}{$\mathrm{Q} X \sum \rightarrow \mathrm{Q}$} & \multicolumn{3}{|c|}{$\mathrm{Q} X \sum \rightarrow \mathrm{Q}$} & \multicolumn{3}{|c|}{$\mathrm{Q} X \sum \rightarrow \mathrm{Q}$} \\
\hline $\mathrm{s}_{0}$ & 1 & $\mathrm{~s}_{2}$ & $\mathrm{~s}_{1}$ & 0 & $\mathrm{~s}_{1}$ & $\mathrm{~s}_{0}$ & 1 & $\mathrm{~s}_{2}$ & $\mathrm{~s}_{3}$ & 0 & $\mathrm{~s}_{3}$ \\
\hline $\mathrm{s}_{2}$ & 0 & $\mathrm{~s}_{1}$ & $\mathrm{~s}_{1}$ & 1 & $\mathrm{~s}_{1}$ & $\mathrm{~S}_{2}$ & 1 & $\mathrm{~s}_{3}$ & $\mathrm{~s}_{3}$ & 1 & $\mathrm{~s}_{3}$ \\
\hline $\mathrm{s}_{1}$ & 0 & $\mathrm{~s}_{1}$ & $\mathrm{~s}_{1}$ & 1 & $\mathrm{~s}_{1}$ & $\mathrm{~s}_{3}$ & 0 & $\mathrm{~s}_{3}$ & $\mathrm{~s}_{3}$ & 0 & $\mathrm{~s}_{3}$ \\
\hline $\mathrm{s}_{1}$ & 0 & $\mathrm{~s}_{1}$ & $\mathrm{~s}_{1}$ & 1 & $\mathrm{~s}_{1}$ & $\mathrm{~s}_{3}$ & 0 & $\mathrm{~s}_{3}$ & $\mathrm{~s}_{3}$ & 0 & $\mathrm{~s}_{3}$ \\
\hline $\mathrm{s}_{1}$ & 1 & $\mathrm{~s}_{1}$ & $\mathrm{~s}_{1}$ & 1 & $\mathrm{~s}_{1}$ & $\mathrm{~s}_{3}$ & 1 & $\mathrm{~s}_{3}$ & $\mathrm{~s}_{3}$ & 0 & $\mathrm{~s}_{3}$ \\
\hline $\mathrm{s}_{1}$ & 1 & $\mathrm{~s}_{1}$ & $\mathrm{~s}_{1}$ & 1 & $\mathrm{~s}_{1}$ & $\mathrm{~s}_{3}$ & 1 & $\mathrm{~s}_{3}$ & $\mathrm{~s}_{3}$ & 1 & $\mathrm{~s}_{3}$ \\
\hline $\mathrm{s}_{1}$ & 1 & $\mathrm{~s}_{1}$ & $\mathrm{~s}_{1}$ & 1 & $\mathrm{~s}_{1}$ & $\mathrm{~s}_{3}$ & 1 & $\mathrm{~s}_{3}$ & $\mathrm{~s}_{3}$ & 0 & $\mathrm{~s}_{3}$ \\
\hline $\mathrm{s}_{1}$ & 1 & $\mathrm{~s}_{1}$ & $\mathrm{~s}_{1}$ & 1 & $\mathrm{~s}_{1}$ & $\mathrm{~s}_{3}$ & 1 & $\mathrm{~s}_{3}$ & $\mathrm{~s}_{3}$ & 1 & $\mathrm{~s}_{3}$ \\
\hline $\mathrm{s}_{1}$ & 0 & $\mathrm{~s}_{1}$ & $\mathrm{~s}_{1}$ & 0 & $\mathrm{~s}_{1}$ & $\mathrm{~s}_{3}$ & 0 & $\mathrm{~s}_{3}$ & $\mathrm{~s}_{3}$ & 0 & $\mathrm{~s}_{3}$ \\
\hline $\mathrm{s}_{1}$ & 1 & $\mathrm{~s}_{1}$ & $\mathrm{~s}_{1}$ & 1 & $\mathrm{~s}_{1}$ & $\mathrm{~s}_{3}$ & 1 & $\mathrm{~s}_{3}$ & $\mathrm{~s}_{3}$ & 1 & $\mathrm{~s}_{3}$ \\
\hline $\mathrm{s}_{1}$ & 1 & $\mathrm{~s}_{1}$ & $\mathrm{~s}_{1}$ & 1 & $\mathrm{~s}_{1}$ & $\mathrm{~S}_{3}$ & 1 & $\mathrm{~s}_{3}$ & $\mathrm{~s}_{3}$ & 1 & $\mathrm{~s}_{3}$ \\
\hline $\mathrm{s}_{1}$ & 0 & $\mathrm{~s}_{1}$ & $\mathrm{~s}_{1}$ & 0 & $\mathrm{~s}_{1}$ & $\mathrm{~s}_{3}$ & 0 & $\mathrm{~s}_{3}$ & $\mathrm{~s}_{3}$ & 0 & $\mathrm{~s}_{3}$ \\
\hline $\mathrm{s}_{1}$ & 1 & $\mathrm{~s}_{1}$ & $\mathrm{~s}_{1}$ & 0 & $\mathrm{~s}_{1}$ & $\mathrm{~s}_{3}$ & 1 & $\mathrm{~s}_{3}$ & $\mathrm{~s}_{3}$ & 0 & $\mathrm{~s}_{3}$ \\
\hline $\mathrm{s}_{1}$ & 1 & $\mathrm{~s}_{1}$ & $\mathrm{~S}_{1}$ & 0 & $\mathrm{~s}_{1}$ & $\mathrm{~S}_{3}$ & 1 & $\mathrm{~s}_{3}$ & $\mathrm{~s}_{3}$ & 1 & $\mathrm{~S}_{3}$ \\
\hline $\mathrm{S}_{1}$ & 1 & $\mathrm{~S}_{1}$ & $\mathrm{~s}_{1}$ & 1 & $\mathrm{~s}_{1}$ & $\mathrm{~s}_{3}$ & 1 & $\mathrm{~s}_{3}$ & $\mathrm{~s}_{3}$ & 1 & $\mathrm{~S}_{3}$ \\
\hline $\mathrm{s}_{1}$ & 1 & $\mathrm{~s}_{1}$ & $\mathrm{~s}_{1}$ & 1 & $\mathrm{~s}_{1}$ & $\mathrm{~S}_{3}$ & 1 & $\mathrm{~s}_{3}$ & $\mathrm{~s}_{3}$ & 0 & $\mathrm{~S}_{3}$ \\
\hline
\end{tabular}


The input string to validate class ' $\mathrm{C}$ ' IP Address:

\begin{tabular}{|c|c|c|c|}
\hline & \multirow{2}{*}{ Table 8.a } & Dotted Decimal Notation & [Should be Accepted] \\
\hline \multirow{3}{*}{$\begin{array}{c}\mathrm{IPV}_{4} \\
\text { Address }\end{array}$} & & Binary Notation & 11001111011011110111111101100011 \\
\hline & \multirow{2}{*}{ Table 8.b } & Dotted Decimal Notation & [Should be Rejected] \\
\hline & & Binary Notation & 01001111011011110100010101100110 \\
\hline
\end{tabular}

In the table 8.a, the last state achieved through the automata is a final state. The automata is verified and hence proved that it accepts all the strings which start with ' 110 'i.e., Class ' $\mathrm{C}$ ' IPV ${ }_{4}$ Address. In the table 8.b, since the last state obtained is $\mathrm{s}_{4}$ and as it is not the final state in the automata, so the strings which start with other than ' 110 ' are not accepted i.e., it accepts only Class ' $C$ ' IPV ${ }_{4}$ Address.

Table 8. Validation table that shows the Automata accepts Class 'C' IPV4 Address

\begin{tabular}{|c|c|c|c|c|c|c|c|c|c|c|c|}
\hline \multicolumn{6}{|c|}{ Table 8.a } & \multicolumn{6}{|c|}{ Table 8.b } \\
\hline $\begin{array}{l}\text { Current } \\
\text { State-Q }\end{array}$ & $\sum_{\sum}^{\text {Input- }}$ & $\begin{array}{c}\text { Next } \\
\text { State- } \\
Q \\
\end{array}$ & $\begin{array}{l}\text { Current } \\
\text { State-Q }\end{array}$ & $\sum_{\sum}^{\text {Input- }}$ & $\begin{array}{c}\text { Next } \\
\text { State- } \\
\text { Q }\end{array}$ & $\begin{array}{l}\text { Current } \\
\text { State-Q }\end{array}$ & $\sum_{\Sigma}^{\text {Input- }}$ & $\begin{array}{c}\text { Next } \\
\text { State- } \\
\text { Q } \\
\end{array}$ & $\begin{array}{l}\text { Current } \\
\text { State-Q }\end{array}$ & $\sum_{\sum}^{\text {Input- }}$ & $\begin{array}{c}\text { Next } \\
\text { State- } \\
Q \\
\end{array}$ \\
\hline \multicolumn{3}{|c|}{$\mathrm{Q} X \sum \rightarrow \mathrm{Q}$} & \multicolumn{3}{|c|}{$\mathrm{Q} X \sum \rightarrow \mathrm{Q}$} & \multicolumn{3}{|c|}{$\mathrm{Q} X \sum \rightarrow \mathrm{Q}$} & \multicolumn{3}{|c|}{$\mathrm{Q} X \sum \rightarrow \mathrm{Q}$} \\
\hline $\mathrm{s}_{0}$ & 1 & $\mathrm{~s}_{3}$ & $\mathrm{~s}_{1}$ & 0 & $\mathrm{~s}_{1}$ & $\mathrm{~s}_{0}$ & 0 & $\mathrm{~S}_{4}$ & $\mathrm{~S}_{4}$ & 0 & $\mathrm{~S}_{4}$ \\
\hline $\mathrm{s}_{3}$ & 1 & $\mathrm{~s}_{2}$ & $\mathrm{~s}_{1}$ & 1 & $\mathrm{~s}_{1}$ & $\mathrm{~s}_{4}$ & 1 & $\mathrm{~S}_{4}$ & $\mathrm{~S}_{4}$ & 1 & $\mathrm{~S}_{4}$ \\
\hline $\mathrm{s}_{2}$ & 0 & $\mathrm{~s}_{1}$ & $\mathrm{~s}_{1}$ & 1 & $\mathrm{~s}_{1}$ & $\mathrm{~s}_{4}$ & 0 & $\mathrm{~s}_{4}$ & $\mathrm{~s}_{4}$ & 0 & $\mathrm{~s}_{4}$ \\
\hline $\mathrm{s}_{1}$ & 0 & $\mathrm{~s}_{1}$ & $\mathrm{~s}_{1}$ & 1 & $\mathrm{~s}_{1}$ & $\mathrm{~s}_{4}$ & 0 & $\mathrm{~s}_{4}$ & $\mathrm{~S}_{4}$ & 0 & $\mathrm{~s}_{4}$ \\
\hline $\mathrm{s}_{1}$ & 1 & $\mathrm{~s}_{1}$ & $\mathrm{~s}_{1}$ & 1 & $\mathrm{~s}_{1}$ & $\mathrm{~s}_{4}$ & 1 & $\mathrm{~s}_{4}$ & $\mathrm{~S}_{4}$ & 0 & $\mathrm{~s}_{4}$ \\
\hline $\mathrm{s}_{1}$ & 1 & $\mathrm{~s}_{1}$ & $\mathrm{~s}_{1}$ & 1 & $\mathrm{~s}_{1}$ & $\mathrm{~S}_{4}$ & 1 & $\mathrm{~S}_{4}$ & $\mathrm{~S}_{4}$ & 1 & $\mathrm{~S}_{4}$ \\
\hline $\mathrm{s}_{1}$ & 1 & $\mathrm{~s}_{1}$ & $\mathrm{~s}_{1}$ & 1 & $\mathrm{~s}_{1}$ & $\mathrm{~S}_{4}$ & 1 & $\mathrm{~S}_{4}$ & $\mathrm{~S}_{4}$ & 0 & $\mathrm{~S}_{4}$ \\
\hline $\mathrm{s}_{1}$ & 1 & $\mathrm{~s}_{1}$ & $\mathrm{~s}_{1}$ & 1 & $\mathrm{~s}_{1}$ & $\mathrm{~S}_{4}$ & 1 & $\mathrm{~S}_{4}$ & $\mathrm{~S}_{4}$ & 1 & $\mathrm{~S}_{4}$ \\
\hline $\mathrm{s}_{1}$ & 0 & $\mathrm{~s}_{1}$ & $\mathrm{~s}_{1}$ & 0 & $\mathrm{~s}_{1}$ & $\mathrm{~S}_{4}$ & 0 & $\mathrm{~S}_{4}$ & $\mathrm{~S}_{4}$ & 0 & $\mathrm{~S}_{4}$ \\
\hline $\mathrm{s}_{1}$ & 1 & $\mathrm{~s}_{1}$ & $\mathrm{~s}_{1}$ & 1 & $\mathrm{~s}_{1}$ & $\mathrm{~S}_{4}$ & 1 & $\mathrm{~s}_{4}$ & $\mathrm{~s}_{4}$ & 1 & $\mathrm{~S}_{4}$ \\
\hline $\mathrm{s}_{1}$ & 1 & $\mathrm{~s}_{1}$ & $\mathrm{~s}_{1}$ & 1 & $\mathrm{~s}_{1}$ & $\mathrm{~S}_{4}$ & 1 & $\mathrm{~s}_{4}$ & $\mathrm{~S}_{4}$ & 1 & $\mathrm{~s}_{4}$ \\
\hline $\mathrm{s}_{1}$ & 0 & $\mathrm{~s}_{1}$ & $\mathrm{~s}_{1}$ & 0 & $\mathrm{~s}_{1}$ & $\mathrm{~s}_{4}$ & 0 & $\mathrm{~S}_{4}$ & $\mathrm{~S}_{4}$ & 0 & $\mathrm{~s}_{4}$ \\
\hline $\mathrm{s}_{1}$ & 1 & $\mathrm{~s}_{1}$ & $\mathrm{~s}_{1}$ & 0 & $\mathrm{~s}_{1}$ & $\mathrm{~s}_{4}$ & 1 & $\mathrm{~S}_{4}$ & $\mathrm{~S}_{4}$ & 0 & $\mathrm{~S}_{4}$ \\
\hline $\mathrm{s}_{1}$ & 1 & $\mathrm{~s}_{1}$ & $\mathrm{~s}_{1}$ & 0 & $\mathrm{~s}_{1}$ & $\mathrm{~S}_{4}$ & 1 & $\mathrm{~S}_{4}$ & $\mathrm{~S}_{4}$ & 1 & $\mathrm{~S}_{4}$ \\
\hline $\mathrm{s}_{1}$ & 1 & $\mathrm{~s}_{1}$ & $\mathrm{~s}_{1}$ & 1 & $\mathrm{~s}_{1}$ & $\mathrm{~S}_{4}$ & 1 & $\mathrm{~S}_{4}$ & $\mathrm{~S}_{4}$ & 1 & $\mathrm{~S}_{4}$ \\
\hline $\mathrm{s}_{1}$ & 1 & $\mathrm{~s}_{1}$ & $\mathrm{~s}_{1}$ & 1 & $\mathrm{~s}_{1}$ & $\mathrm{~S}_{4}$ & 1 & $\mathrm{~S}_{4}$ & $\mathrm{~S}_{4}$ & 0 & $\mathrm{~S}_{4}$ \\
\hline
\end{tabular}


The input string to validate class ' $\mathrm{D}$ ' IP Address:

\begin{tabular}{|c|c|c|c|}
\hline & \multirow{2}{*}{ Table 9.a } & Dotted Decimal Notation & [Should be Accepted] \\
\hline \multirow{3}{*}{$\begin{array}{c}\mathrm{IPV}_{4} \\
\text { Address }\end{array}$} & & Binary Notation & 11101111011011110111111101100011 \\
\hline & \multirow{2}{*}{ Table 9.b } & Dotted Decimal Notation & [Should be Rejected] \\
\hline & & Binary Notation & 11001010010001010010101010101011 \\
\hline
\end{tabular}

In the table 9.a, the last state achieved through the automata is a final state. The automata is verified and hence proved that it accepts all the strings which start with '1110'i.e., Class 'D' IPV 4 Address. In the table 9.b, since the last state obtained is $s_{5}$ and as it is not the final state in the automata, so the strings which start with other than '1110' are not accepted i.e., it accepts only Class 'D' IPV 4 Address.

Table 9. Validation table that shows the Automata accepts Class 'D' IPV4 Address

\begin{tabular}{|c|c|c|c|c|c|c|c|c|c|c|c|}
\hline \multicolumn{6}{|c|}{ Table 9.a } & \multicolumn{6}{|c|}{ Table 9.b } \\
\hline $\begin{array}{l}\text { Current } \\
\text { State-Q }\end{array}$ & $\sum^{\text {Input- }}$ & $\begin{array}{l}\text { Next } \\
\text { State-Q }\end{array}$ & $\begin{array}{l}\text { Current } \\
\text { State-Q }\end{array}$ & $\sum^{\text {Input- }}$ & $\begin{array}{c}\text { Next } \\
\text { State-Q }\end{array}$ & $\begin{array}{l}\text { Current } \\
\text { State-Q }\end{array}$ & $\sum^{\text {Input- }}$ & $\begin{array}{c}\text { Next } \\
\text { State-Q }\end{array}$ & $\begin{array}{l}\text { Current } \\
\text { State-Q } \\
\end{array}$ & $\begin{array}{l}\text { Input- } \\
\sum\end{array}$ & $\begin{array}{c}\text { Next } \\
\text { State-Q }\end{array}$ \\
\hline \multicolumn{3}{|c|}{$\mathrm{Q} X \sum \rightarrow \mathrm{Q}$} & \multicolumn{3}{|c|}{$\mathrm{Q} X \sum \rightarrow \mathrm{Q}$} & \multicolumn{3}{|c|}{$\mathrm{Q} X \sum \rightarrow \mathrm{Q}$} & \multicolumn{3}{|c|}{$\mathrm{Q} X \sum \rightarrow \mathrm{Q}$} \\
\hline $\mathrm{s}_{0}$ & 1 & $\mathrm{~s}_{4}$ & $\mathrm{~s}_{1}$ & 0 & $\mathrm{~s}_{1}$ & $\mathrm{~s}_{0}$ & 1 & $\mathrm{~s}_{4}$ & $\mathrm{~s}_{5}$ & 0 & $\mathrm{~S}_{5}$ \\
\hline $\mathrm{s}_{4}$ & 1 & $\mathrm{~s}_{3}$ & $\mathrm{~s}_{1}$ & 1 & $\mathrm{~s}_{1}$ & $\mathrm{~s}_{4}$ & 1 & $\mathrm{~s}_{3}$ & $\mathrm{~s}_{5}$ & 0 & $\mathrm{~s}_{5}$ \\
\hline $\mathrm{S}_{3}$ & 1 & $\mathrm{~S}_{2}$ & $\mathrm{~s}_{1}$ & 1 & $\mathrm{~s}_{1}$ & $\mathrm{~S}_{3}$ & 0 & $\mathrm{~S}_{5}$ & $\mathrm{~S}_{5}$ & 1 & $\mathrm{~S}_{5}$ \\
\hline $\mathrm{s}_{2}$ & 0 & $\mathrm{~s}_{1}$ & $\mathrm{~s}_{1}$ & 1 & $\mathrm{~s}_{1}$ & $\mathrm{~S}_{5}$ & 0 & $\mathrm{~S}_{5}$ & $\mathrm{~S}_{5}$ & 0 & $\mathrm{~S}_{5}$ \\
\hline $\mathrm{s}_{1}$ & 1 & $\mathrm{~s}_{1}$ & $\mathrm{~s}_{1}$ & 1 & $\mathrm{~s}_{1}$ & $\mathrm{~s}_{5}$ & 1 & $\mathrm{~S}_{5}$ & $\mathrm{~S}_{5}$ & 1 & $\mathrm{~S}_{5}$ \\
\hline $\mathrm{s}_{1}$ & 1 & $\mathrm{~s}_{1}$ & $\mathrm{~s}_{1}$ & 1 & $\mathrm{~s}_{1}$ & $\mathrm{~S}_{5}$ & 0 & $\mathrm{~S}_{5}$ & $\mathrm{~s}_{5}$ & 0 & $\mathrm{~s}_{5}$ \\
\hline $\mathrm{s}_{1}$ & 1 & $\mathrm{~s}_{1}$ & $\mathrm{~s}_{1}$ & 1 & $\mathrm{~s}_{1}$ & $\mathrm{~S}_{5}$ & 1 & $\mathrm{~S}_{5}$ & $\mathrm{~S}_{5}$ & 1 & $\mathrm{~S}_{5}$ \\
\hline $\mathrm{s}_{1}$ & 1 & $\mathrm{~s}_{1}$ & $\mathrm{~s}_{1}$ & 1 & $\mathrm{~s}_{1}$ & $\mathrm{~s}_{5}$ & 0 & $\mathrm{~s}_{5}$ & $\mathrm{~S}_{5}$ & 0 & $\mathrm{~s}_{5}$ \\
\hline $\mathrm{s}_{1}$ & 0 & $\mathrm{~s}_{1}$ & $\mathrm{~s}_{1}$ & 0 & $\mathrm{~s}_{1}$ & $\mathrm{~s}_{5}$ & 0 & $\mathrm{~S}_{5}$ & $\mathrm{~S}_{5}$ & 1 & $\mathrm{~s}_{5}$ \\
\hline $\mathrm{s}_{1}$ & 1 & $\mathrm{~s}_{1}$ & $\mathrm{~s}_{1}$ & 1 & $\mathrm{~s}_{1}$ & $\mathrm{~s}_{5}$ & 1 & $\mathrm{~s}_{5}$ & $\mathrm{~S}_{5}$ & 0 & $\mathrm{~s}_{5}$ \\
\hline $\mathrm{s}_{1}$ & 1 & $\mathrm{~s}_{1}$ & $\mathrm{~s}_{1}$ & 1 & $\mathrm{~s}_{1}$ & $\mathrm{~s}_{5}$ & 0 & $\mathrm{~s}_{5}$ & $\mathrm{~S}_{5}$ & 1 & $\mathrm{~s}_{5}$ \\
\hline $\mathrm{s}_{1}$ & 0 & $\mathrm{~s}_{1}$ & $\mathrm{~s}_{1}$ & 0 & $\mathrm{~s}_{1}$ & $\mathrm{~S}_{5}$ & 0 & $\mathrm{~S}_{5}$ & $\mathrm{~s}_{5}$ & 0 & $\mathrm{~s}_{5}$ \\
\hline $\mathrm{s}_{1}$ & 1 & $\mathrm{~s}_{1}$ & $\mathrm{~s}_{1}$ & 0 & $\mathrm{~s}_{1}$ & $\mathrm{~S}_{5}$ & 0 & $\mathrm{~S}_{5}$ & $\mathrm{~S}_{5}$ & 1 & $\mathrm{~S}_{5}$ \\
\hline $\mathrm{s}_{1}$ & 1 & $\mathrm{~s}_{1}$ & $\mathrm{~s}_{1}$ & 0 & $\mathrm{~s}_{1}$ & $\mathrm{~S}_{5}$ & 1 & $\mathrm{~S}_{5}$ & $\mathrm{~S}_{5}$ & 0 & $\mathrm{~s}_{5}$ \\
\hline $\mathrm{s}_{1}$ & 1 & $\mathrm{~s}_{1}$ & $\mathrm{~s}_{1}$ & 1 & $\mathrm{~s}_{1}$ & $\mathrm{~S}_{5}$ & 0 & $\mathrm{~S}_{5}$ & $\mathrm{~S}_{5}$ & 1 & $\mathrm{~s}_{5}$ \\
\hline $\mathrm{s}_{1}$ & 1 & $\mathrm{~s}_{1}$ & $\mathrm{~s}_{1}$ & 1 & $\mathrm{~s}_{1}$ & $\mathrm{~s}_{5}$ & 1 & $\mathrm{~S}_{5}$ & $\mathrm{~S}_{5}$ & 1 & $\mathrm{~s}_{5}$ \\
\hline
\end{tabular}


The input string to validate class 'E' IP Address:

\begin{tabular}{|c|c|c|c|}
\hline & \multirow{2}{*}{ Table 10.a } & Dotted Decimal Notation & [Should be Accepted] \\
\hline \multirow{3}{*}{$\begin{array}{c}\mathrm{IPV}_{4} \\
\text { Address }\end{array}$} & & Binary Notation & 11110101010101010101001010101010 \\
\hline & \multirow{2}{*}{ Table 10.b } & Dotted Decimal Notation & [Should be Rejected] \\
\hline & & Binary Notation & 10101110100111101010001010101110 \\
\hline
\end{tabular}

In the table 10.a, the last state achieved through the automata is a final state. The automata is verified and hence proved that it accepts all the strings which start with ' 1111 'i.e., Class ' $E$ ' IPV ${ }_{4}$ Address. In the table 10.b, since the last state obtained is $s_{3}$ and as it is not the final state in the automata, so the strings which start with other than ' 1111 ' are not accepted i.e., it accepts only Class ' $E$ ' $\mathrm{IPV}_{4}$ Address.

Table 10. Validation table that shows the Automata accepts Class 'E' IPV4 Address

\begin{tabular}{|c|c|c|c|c|c|c|c|c|c|c|c|}
\hline \multicolumn{6}{|c|}{ Table 10.a } & \multicolumn{6}{|c|}{ Table 10.b } \\
\hline $\begin{array}{l}\text { Current } \\
\text { State-Q }\end{array}$ & $\begin{array}{l}\text { Input- } \\
\sum\end{array}$ & $\begin{array}{c}\text { Next } \\
\text { State-Q }\end{array}$ & $\begin{array}{l}\text { Current } \\
\text { State-Q }\end{array}$ & $\sum^{\text {Input- }}$ & $\begin{array}{c}\text { Next } \\
\text { State-Q }\end{array}$ & $\begin{array}{l}\text { Current } \\
\text { State-Q }\end{array}$ & $\sum^{\text {Input- }}$ & $\begin{array}{c}\text { Next } \\
\text { State-Q }\end{array}$ & $\begin{array}{l}\text { Current } \\
\text { State-Q }\end{array}$ & $\sum^{\text {Input- }}$ & $\begin{array}{c}\text { Next } \\
\text { State-Q }\end{array}$ \\
\hline \multicolumn{3}{|c|}{$\mathrm{Q} X \quad \sum \rightarrow \mathrm{Q}$} & \multicolumn{3}{|c|}{$\mathrm{Q} X \sum \rightarrow \mathrm{Q}$} & \multicolumn{3}{|c|}{$\mathrm{Q} X \sum \rightarrow \mathrm{Q}$} & \multicolumn{3}{|c|}{$\mathrm{Q} X \sum \rightarrow \mathrm{Q}$} \\
\hline $\mathrm{s}_{0}$ & 1 & $\mathrm{~s}_{4}$ & $\mathrm{~s}_{1}$ & 0 & $\mathrm{~s}_{1}$ & $\mathrm{~s}_{0}$ & 1 & $\mathrm{~s}_{4}$ & $\mathrm{~S}_{5}$ & 1 & $\mathrm{~s}_{5}$ \\
\hline $\mathrm{s}_{4}$ & 1 & $\mathrm{~s}_{3}$ & $\mathrm{~s}_{1}$ & 1 & $\mathrm{~s}_{1}$ & $\mathrm{~s}_{4}$ & 0 & $\mathrm{~S}_{5}$ & $\mathrm{~S}_{5}$ & 0 & $\mathrm{~s}_{5}$ \\
\hline $\mathrm{s}_{3}$ & 1 & $\mathrm{~s}_{2}$ & $\mathrm{~s}_{1}$ & 0 & $\mathrm{~s}_{1}$ & $\mathrm{~s}_{5}$ & 1 & $\mathrm{~s}_{5}$ & $\mathrm{~s}_{5}$ & 1 & $\mathrm{~s}_{5}$ \\
\hline $\mathrm{s}_{2}$ & 1 & $\mathrm{~s}_{1}$ & $\mathrm{~s}_{1}$ & 1 & $\mathrm{~s}_{1}$ & $\mathrm{~S}_{5}$ & 0 & $\mathrm{~s}_{5}$ & $\mathrm{~s}_{5}$ & 0 & $\mathrm{~s}_{5}$ \\
\hline $\mathrm{s}_{1}$ & 0 & $\mathrm{~s}_{1}$ & $\mathrm{~s}_{1}$ & 0 & $\mathrm{~s}_{1}$ & $\mathrm{~s}_{5}$ & 1 & $\mathrm{~s}_{5}$ & $\mathrm{~s}_{5}$ & 0 & $\mathrm{~s}_{5}$ \\
\hline $\mathrm{s}_{1}$ & 1 & $\mathrm{~s}_{1}$ & $\mathrm{~s}_{1}$ & 0 & $\mathrm{~s}_{1}$ & $\mathrm{~S}_{5}$ & 1 & $\mathrm{~s}_{5}$ & $\mathrm{~s}_{5}$ & 0 & $\mathrm{~s}_{5}$ \\
\hline $\mathrm{s}_{1}$ & 0 & $\mathrm{~s}_{1}$ & $\mathrm{~s}_{1}$ & 1 & $\mathrm{~s}_{1}$ & $\mathrm{~s}_{5}$ & 1 & $\mathrm{~s}_{5}$ & $\mathrm{~S}_{5}$ & 1 & $\mathrm{~s}_{5}$ \\
\hline $\mathrm{s}_{1}$ & 1 & $\mathrm{~s}_{1}$ & $\mathrm{~s}_{1}$ & 0 & $\mathrm{~s}_{1}$ & $\mathrm{~s}_{5}$ & 0 & $\mathrm{~s}_{5}$ & $\mathrm{~s}_{5}$ & 0 & $\mathrm{~s}_{5}$ \\
\hline $\mathrm{s}_{1}$ & 0 & $\mathrm{~s}_{1}$ & $\mathrm{~s}_{1}$ & 1 & $\mathrm{~s}_{1}$ & $\mathrm{~s}_{5}$ & 1 & $\mathrm{~s}_{5}$ & $\mathrm{~s}_{5}$ & 1 & $\mathrm{~s}_{5}$ \\
\hline $\mathrm{s}_{1}$ & 1 & $\mathrm{~s}_{1}$ & $\mathrm{~s}_{1}$ & 0 & $\mathrm{~s}_{1}$ & $\mathrm{~s}_{5}$ & 0 & $\mathrm{~s}_{5}$ & $\mathrm{~S}_{5}$ & 0 & $\mathrm{~s}_{5}$ \\
\hline $\mathrm{s}_{1}$ & 0 & $\mathrm{~s}_{1}$ & $\mathrm{~s}_{1}$ & 1 & $\mathrm{~s}_{1}$ & $\mathrm{~S}_{5}$ & 0 & $\mathrm{~S}_{5}$ & $\mathrm{~S}_{5}$ & 1 & $\mathrm{~S}_{5}$ \\
\hline $\mathrm{s}_{1}$ & 1 & $\mathrm{~s}_{1}$ & $\mathrm{~s}_{1}$ & 0 & $\mathrm{~s}_{1}$ & $\mathrm{~S}_{5}$ & 1 & $\mathrm{~s}_{5}$ & $\mathrm{~S}_{5}$ & 0 & $\mathrm{~s}_{5}$ \\
\hline $\mathrm{s}_{1}$ & 0 & $\mathrm{~s}_{1}$ & $\mathrm{~s}_{1}$ & 1 & $\mathrm{~s}_{1}$ & $\mathrm{~s}_{5}$ & 1 & $\mathrm{~s}_{5}$ & $\mathrm{~s}_{5}$ & 1 & $\mathrm{~s}_{5}$ \\
\hline $\mathrm{s}_{1}$ & 1 & $\mathrm{~s}_{1}$ & $\mathrm{~s}_{1}$ & 0 & $\mathrm{~s}_{1}$ & $\mathrm{~s}_{5}$ & 1 & $\mathrm{~s}_{5}$ & $\mathrm{~s}_{5}$ & 1 & $\mathrm{~s}_{5}$ \\
\hline $\mathrm{s}_{1}$ & 0 & $\mathrm{~s}_{1}$ & $\mathrm{~s}_{1}$ & 1 & $\mathrm{~s}_{1}$ & $\mathrm{~S}_{5}$ & 1 & $\mathrm{~s}_{5}$ & $\mathrm{~S}_{5}$ & 1 & $\mathrm{~s}_{5}$ \\
\hline $\mathrm{s}_{1}$ & 1 & $\mathrm{~s}_{1}$ & $\mathrm{~s}_{1}$ & 0 & $\mathrm{~s}_{1}$ & $\mathrm{~s}_{5}$ & 0 & $\mathrm{~S}_{5}$ & $\mathrm{~s}_{5}$ & 0 & $\mathrm{~S}_{5}$ \\
\hline
\end{tabular}




\section{Conclusions}

Any problem in the Computer Science can be virtualized in terms of the abstract machines viz., Finite Automata. Thus we have discussed the design of Automata that accepts the class of IP addresses. The proven validations in the results section states that the designed automata accept the corresponding class of IPV Addresses.

\section{References}

[1] J.E.Hopcraft and Jeffery D.Ulman: Introduction to Automata Theory, Languages \& Computation byNarosa Publishing Company.

[2] Mishra \&Chandra Sekharan: Theory of Computer Science PHI.

[3] Peter Linz: An Introduction to Formal Languages and Automata, 3e by- Narosa Publishing House.

[4] Sipser: Introduction to Theory of Computation, 2/e, Thomson.

[5] Daniel I.A. Cohen: Introduction to Computer Theory, John Wiley.

[6] Behrouz A.Forouzan, Data Communications and Networking, $3^{\text {rd }}$ EditionTMH,2004

[7] William Stallings, Data and Computer Communications, $7^{\text {th }}$ Edition, Pearson Education Inc., 2004.

[8] Martin JC. Introduction to languages and the theory of computation. $3^{\text {rd }}$ edition Tata Mcgraw Hill; 2010.

[9] Saradhi Varma G.P, Tirupathi Rao B, Theory of Computation-Formal Languages and Automata Theory, Scitech Publications.

[10] P. Ezhilarasu, J. Prakash, N. Krishnaraj, D. Satheesh Kumar, K. Suresh Babu and C. Parthasarathy, A Novel Approach to Design the Finite Automata to Accept the Palindrome with the Three Input Characters, Indian Journal of Science and Technology, Vol 8(28), DOI: 10.17485/ijst/2015/v8i28/78189, October 2015.

[11] Ezhilarasu P, Krishnaraj N. Triple Substring Based Classification for Nondeterministic Finite Automata. IJAER. 2015; 10(59):177-82.

[12] Daniel I.A. Cohen, Introduction to Computer Theory, $2^{\text {nd }}$ edition, Wiley Publishers 2007.

[13] S.N. Sivanandam, M. Janaki Meena, Theory of Computation, Published by I.K. International Publishing House Pvt. Ltd.

[14] MARTIN (Author), Introduction To Languages And The Theory Of Computation (), $3^{\text {rd }}$ Edition

[15] S.R. Jena, S.K. Swain, Theory of Computation and Application-2017.

\section{Authors' Profiles}

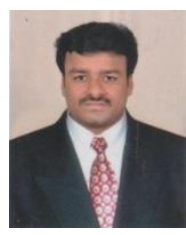

Sri Ram Chandra. $\mathbf{P}$ has received his Bachelor's Degree in Computer science and Engineering from Andhra University, Master's Degree from GITAM University in the years 2010 and 2012 respectively. Currently he is a researcher in GITAM University with Theory of Computation and Cryptosystems Design \& Cryptanalysis as research interests. At present he is working as Associate Professor in the department of Computer Science and Engineering, Godavari Institute of Engineering and Technology (A), Rajahmundry, Andhra Pradesh, INDIA. He is a member of CSI. He has published 03 research papers in reputed International journals. He has 6.4 Years of Teaching Experience. 


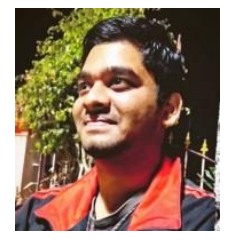

K.S.Sravan is pursuing his Bachelor's Degree in Computer science and Engineering from Godavari Institute of Engineering and Technology (A), Rajahmundry, Andhra Pradesh, INDIA. He is in interested in Theory of Computations, Databases.

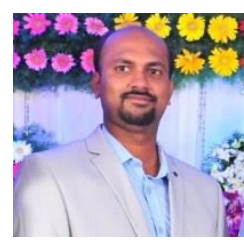

Srinivas Chakravarthy $\mathbf{M}$ has received his Bachelor's Degree, Master's Degree in Computer science and Engineering from JNTU-Kakinada in the years 2008 and 2011 respectively. Currently he is a researcher in GITAM University with Network Security as research interests. At present he is working as Associate Professor in the department of Computer Science and Engineering, Godavari Institute of Engineering and Technology (A), Rajahmundry, Andhra Pradesh, INDIA.

How to cite this paper: P.Sri Ram Chandra, K.S.Sravan, M.S.Chakravarthy,"A New Approach to the Design of a Finite Automaton that accepts Class of $\mathrm{IPV}_{4}$ Addresses", International Journal of Mathematical Sciences and Computing(IJMSC), Vol.5, No.1, pp.65-79, 2019.DOI: 10.5815/ijmsc.2019.01.06 